\title{
AFM Analysis of Gas Cluster Ion Impact Craters and Smoothing
}

\author{
Christopher Santeufemio*
}

*Epion Corporation, 37 Manning Road, Billerica, MA 01821

AFM analysis indicates that gas cluster ion beam (GCIB) technology could change the way substrate surfaces are processed today. The present commercial application for GCIB is atomic level smoothing of substrate surfaces of a variety of materials, including semiconductors, metals, and photonics materials. This is done with long exposures to the cluster beam in controlled patterns.

Smoothing application exposures were done on a variety of materials. Low dose exposures were done on pristine oxide $\left(\mathrm{SiO}_{2}\right)$ films on silicon wafer surfaces. All AFM data is from a Digital Instruments Dimension 3100 AFM using Tapping Mode, using the highest quality Si AFM probes.

Figure 1 shows two AFM scans of individual cluster impact craters on $\mathrm{SiO}_{2}$ films, which are produced with only a very brief exposure of ionized argon clusters. The craters indicate the removal of material [1]. These craters range in size from $5 \mathrm{~nm}$ to $25 \mathrm{~nm}$ in diameter and $1 \mathrm{~nm}$ to $5 \mathrm{~nm}$ deep.

Figure 2 shows AFM data for lead zirconium titanate (PZT) processed for a smoothing application. Figure 2a shows a $1 \mu$ scan of PZT unprocessed, $2 \mathrm{~b}$ shows the PZT after GCIB processing, and 2c shows the power spectral density (PSD) comparison of $2 \mathrm{a}$ and $2 \mathrm{~b}$. Analysis of the PSD indicates that the long wavelength roughness (sub-micron features) is significantly reduced by GCIB processing. After GCIB exposure, the surface roughness is dominated by a homogeneous short wavelength roughness, which is the result of multiple overlapping craters. This short wavelength roughness is made up of nanometer scale features, as indicated in Figure 2c by the "knee" of the PSD of the GCIB processed sample. The dimensions here are consistent with those of individual craters seen in Fig.1. Analysis of this data shows GCIB's effective smoothing window on the PSD as being between approximately $1 \mu$ and $5 \mathrm{~nm}$. This trend is maintained on features near $1 \mu$ as seen on larger scans [2]. The data indicate that GCIB can modify a rough surface with large $(1 \mu)$ features to a smooth surface with small $(1-5 \mathrm{~nm})$ features [3].

GCIB exposure on $\mathrm{SiO}_{2}$ produces impact craters that have a particular morphology. Through atomic force microscopy and analysis, the morphology of the craters is shown to determine the minimum wavelength roughness of a surface in the smoothing application. The range of crater size is shownto be a dominant wavelength band in the PSD of the fully processed material. GCIB achieves its goal of material removal and surface smoothing by the repeated and combined effects of multiple overlapping cluster impact craters [4].

[1] Yamada et al, Materials Processing by Gas Cluster Ion Beams, Materials Science and Engineering Reports, Vol. R34, No.6 (2001) 231.

[2] L.P. Allen et al., "Substrate Smoothing Using Gas Cluster Ion Beam Processing", Journal of Electronic Materials, Vol. 30, No.7 (2001) 829.

[3] D.B. Fenner et al., "Ion Beam Nano-Smoothing of Sapphire and Silicon Carbide Surfaces" Proceedings of SPIE, Vol. 4468 (2001) 17.

[4] Special thanks to Dr. David Fenner and to Dr. Lisa Allen for invaluable discussion. 


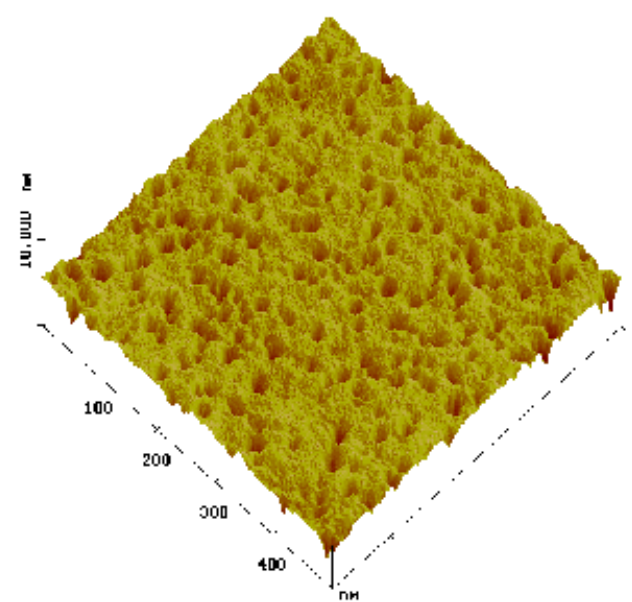

Fig.1a. Argon cluster impacts on $\mathrm{SiO}_{2}$ $500 \times 500 \mathrm{~nm}$ scan $10 \mathrm{~nm} \mathrm{Z} \mathrm{scale}$

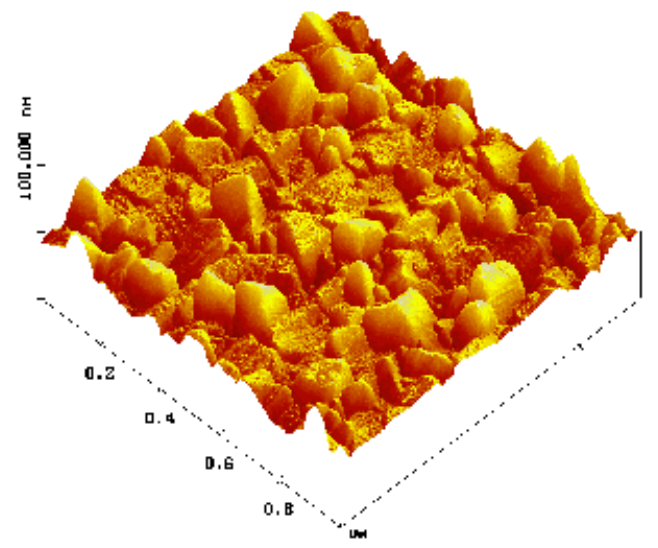

Fig.2a. Unprocessed PZT

$\operatorname{Ra} 43 \AA \quad R m s 56 \AA \quad Z$ range $455 \AA$ $1 \times 1 \mu$ scan $100 \mathrm{~nm} \mathrm{Z}$ scale

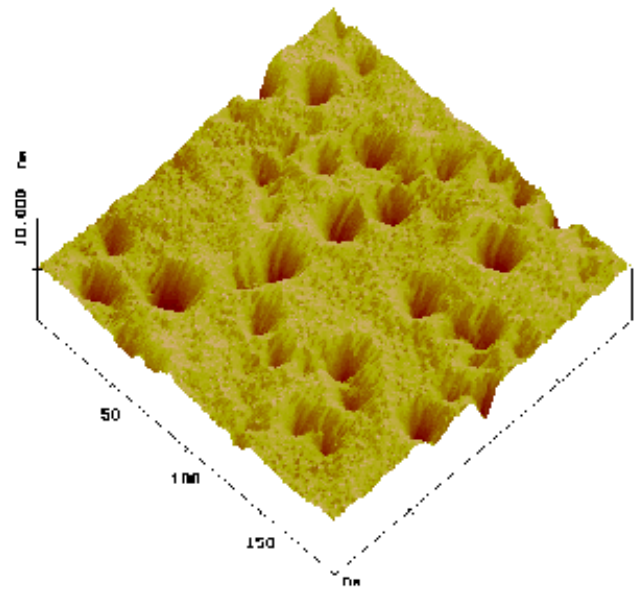

Fig.1b. Argon cluster impacts on $\mathrm{SiO}_{2}$ $200 \times 200 \mathrm{~nm}$ scan $10 \mathrm{~nm} \mathrm{Z}$ scale

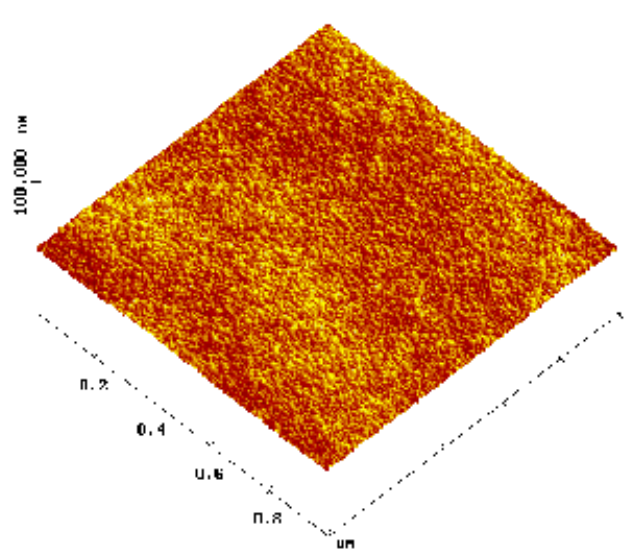

Fig.2b. GCIB Processed PZT

Ra $4 \AA \quad R m s ~ 6 \AA \quad Z$ range $53 \AA$ $1 \times 1 \mu$ scan $100 \mathrm{~nm} \mathrm{Z}$ scale

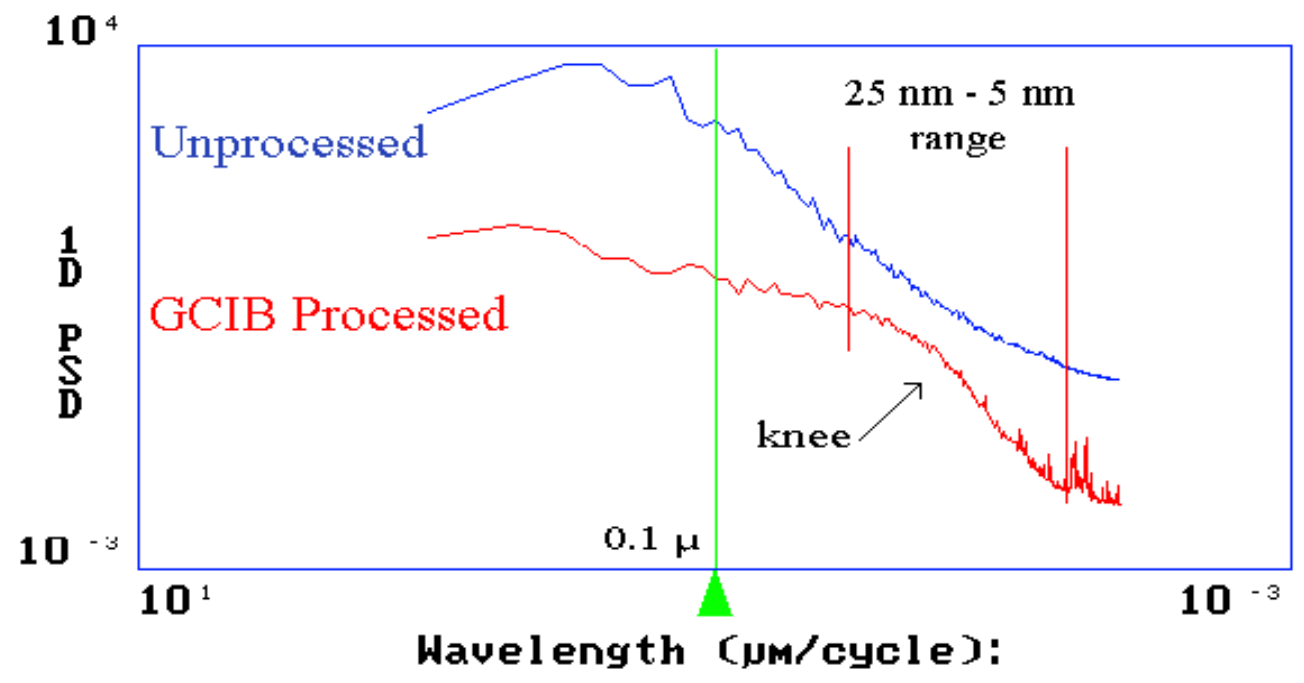

Fig.2c. Power Spectral Density comparison for PZT samples 